\title{
Presencia de neonatos y juveniles del tiburón martillo Sphyrna lewini (Griffith \& Smith, 1834) y del tiburón sedoso Carcharhinus falciformis (Müller \& Henle, 1839) en la costa de Oaxaca, México
}

\author{
Presence of neonates and juvenile scalloped hammerhead sharks Sphyrna lewini (Griffith \& Smith, \\ 1834) and silky sharks Carcharhinus falciformis (Müller \& Henle, 1839) in the Oaxaca coast, \\ Mexico \\ Carmen Alejo-Plata ${ }^{1}$, José L. Gómez-Márquez ${ }^{2}$, Samuel Ramos ${ }^{1}$ y Eduardo Herrera ${ }^{1}$ \\ ${ }^{1}$ Universidad del Mar. Campus Puerto Ángel, Ciudad Universitaria, Puerto Ángel, Distrito de San Pedro Pochutla, Oaxaca, México. \\ AP. 47. CP 70902. (958) 5-84- 30- 78 (T/F) \\ ${ }^{2}$ FES Zaragoza, Universidad Nacional Autónoma de México \\ plata@angel.umar.mx
}

\begin{abstract}
The Oaxaca coast in Mexico has a narrow continental shelf and it reaches depths greater than 500 meters near the coast; furthermore it consists of bays and sheltered areas, where high food availability is found. Each week, from March 2001 to December 2005, the unloading areas of artisanal fisheries between the bays of Huatulco and Puerto Escondido in the Oaxaca coast were visited. Data on fishing gear and zones, as well as the species composition of the captures were taken. In the zone, three fishery units operated: shark, pelagic and demersal. All three capture sharks in a direct or incidental way. At the studied region, 419 organisms of S. lewini were registered; from August to September newborn individuals (43.9\%, 44 to $64 \mathrm{~cm}$ of total length), young of the year (9.3\% $65-75 \mathrm{~cm} \mathrm{Tl})$ and juveniles (30.3\%, 75 to $168 \mathrm{~cm} \mathrm{Tl}$ ) were observed; pregnant females were registered in March, May and June with a fecundity
\end{abstract}

ranging from 18 to 24 embryos ( 35 to $45 \mathrm{~cm} \mathrm{Tl}$ ). With respect to C. falciformis, 1314 individuals were registered. Newborns $(4.7 \%, 65-71 \mathrm{~cm} \mathrm{Tl})$ and young of the year (3.8\%, 72 to 80 $\mathrm{cm} \mathrm{Tl}$ ) were observed from January to September; juveniles (65.4\%, 80 to $170 \mathrm{~cm} \mathrm{Tl)}$ were captured throughout the year; from January to November, 52 pregnant females were observed with embryos at different developmental stages. The presence of newborns, young of the year and pregnant females with embryos in advanced development condition, suggest that the coastal waters near the shoreline of the Oaxaca coast are a spawning and nursery area for these shark species.

Key words: shark, newborns, young of the year, nursing area, Pacific

\section{Introducción}

Los tiburones, por lo general, son organismos muy móviles con una compleja y amplia distribución, particularmente las especies de gran tamaño. Con base en observaciones sobre la distribución de los tiburones por talla y sexo, así como sobre sus patrones de migración, Castro (1993) menciona que se han identificado tres tipos de áreas, de acuerdo al uso de cada una de ellas: 1) áreas de alimentación de adultos, 2) áreas de reproducción y 3) áreas de crianza. Asimismo, se ha documentado que las hembras de muchas especies de tiburón viajan a lugares específicos, geográficamente discretos, para depositar sus huevos o dar a luz a sus crías (Springer 1967); estas áreas pueden ser abiertas o cerradas y son usualmente zonas costeras poco profundas y de alta productividad, en donde los recién nacidos encuentran alimento y protección de sus depredadores, incluyendo a los tiburones adultos (Castro 1993, Simpfendorfer \& Milward 1993, Bonfil 1997, Carlson 1999, Carlson \& Brusher 1999, Hazin et al. 2001, Merson \& Pratt 2001, Costantini \& Affronte 2003, Conrath \& Musick 2007). Estas áreas pueden ser detectadas mediante la observación sistemática y periódica de las hembras grávidas y por la alta proporción de neonatos y juveniles del año (Bonfil 1997).

En las costas de México se han localizado áreas de nacimiento y crianza de varias especies de tiburón, en el Golfo de México y Mar Caribe, se han registrado para Rhizoprionodon terraenovae, Carcharhinus 
acronotus, C. falciformis, C. limbatus y Sphyrna tiburo (Bonfil 1997, Castillo et al. 1998). En el Océano Pacífico, las aguas del noroeste se han reportado como zonas de nacimiento y crianza de Sphyrna lewini, S. zygaena, Carcharhinus limbatus y Rhizoprionodon longurio (Saucedo et al. 1982, Compagno et al. 1995). Para la región del Pacífico central, Anislado (2000) señala como una potencial área de crianza de $S$. lewini a la costa michoacana, debido a la presencia de hembras grávidas, neonatos y juveniles. En el Pacífico sur, a pesar de la importancia que tiene este recurso desde el punto de vista biológico y socioeconómico, los estudios son escasos y generales y con el inconveniente de que consisten en trabajos inéditos. Los antecedentes publicados para esta zona son los reportados por AlejoPlata et al. (2006a y b), mismos que integran información de varios trabajos inéditos.

La mayoría de las publicaciones citadas están orientadas hacia la composición, métodos y artes de pesca de las capturas de tiburón provenientes de la pesca artesanal en los estados de Oaxaca y Chiapas; por lo que en el presente estudio se analiza la presencia de neonatos y juveniles de Carcharhinus falciformis (Müller \& Henle, 1839) y Sphyrna lewini (Griffith \& Smith, 1834), así como de hembras grávidas en diferentes estados de desarrollo, como evidencias de la utilización de la costa de Oaxaca, México, como potencial área de nacimiento y crianza de tiburones de estas especies.

\section{Material y métodos}

\section{Área de estudio}

El área de estudio se localiza dentro de la región conocida como la costa chica de Oaxaca, México (Fig. 1), la cual tiene una extensión aproximada de 280 kilómetros entre Punta Galera y Bahías de Huatulco. La mayor parte de los cambios de temperatura en esta zona se dan de manera anual debido a que sólo hay dos épocas climáticas, secas (noviembre-abril) y lluviosas (mayo-octubre) (Trasviña \& Barton 1997).

\section{Recolecta de datos}

De marzo de 2001 a diciembre de 2005 se visitaron semanalmente los sitios de desembarque de la flota artesanal entre Bahías de Huatulco y Puerto Escondido, en el estado de Oaxaca. Se tomaron datos sobre artes y zona de pesca, así como de la composición de las capturas por especie. La identificación taxonómica se realizó mediante la utilización de claves especializadas (Garrick 1982, Castro 1983, Compagno 1984, Compagno et al. 1995, Castro 2000, Grace 2001) y a cada organismo se le registró la longitud total (Lt) y el sexo. La asignación del estado de desarrollo ontogénico se realizó con base en características descritas por Castro (1993), al considerar a los "neonatos" como organismos con cicatriz umbilical abierta; "juveniles” a organismos sexualmente inmaduros; "machos adultos”

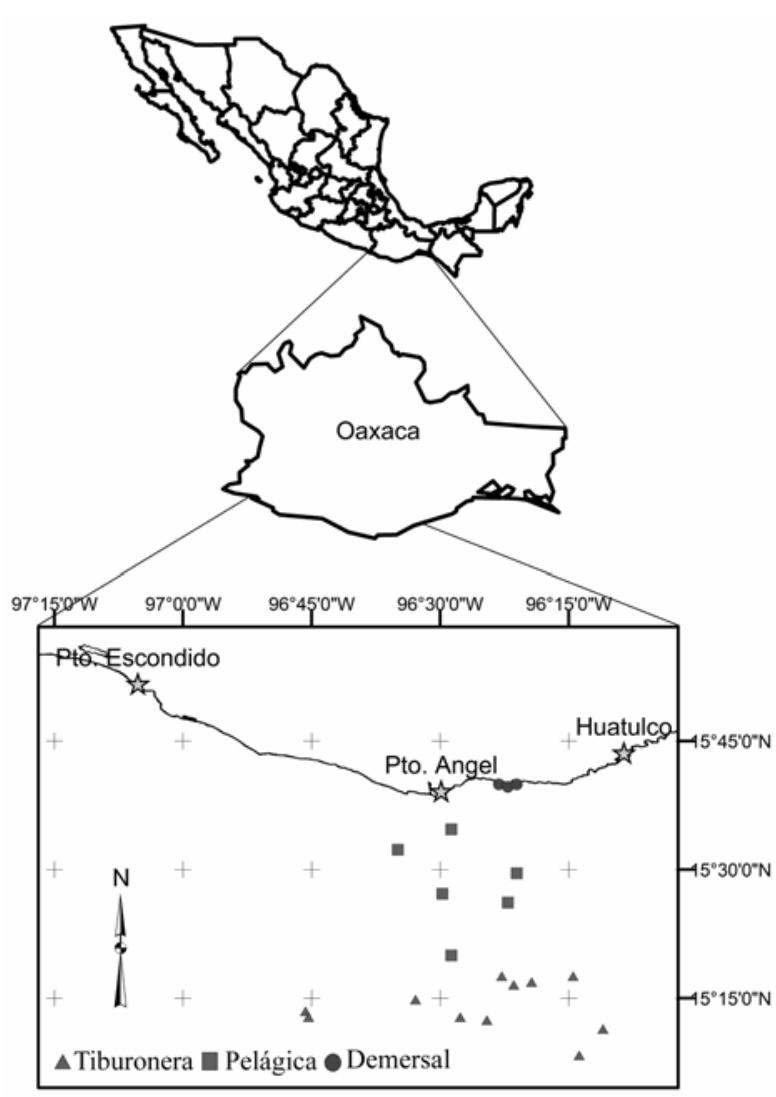

Figura 1

Área de estudio. Zona de pesca artesanal en la costa de Oaxaca, México. Los puntos cercanos a la costa (círculos), representan sitios de pesca con red de enmalle. Los sitios alejados de la costa indican sitios de pesca con palangre modificado (triángulos) y sitios de pesca con palangre tiburonero (cuadrados)

Area of study. Caught area of artisan fishery in Oaxaca, Mexico. Inshore points (circles) represent gill net locations. Offshore points are modified small long lines (triangles) and small long lines locations (squares) 
cuando los mixopterigios se encontraban completamente calcificados y su base podía girar; "hembras maduras" cuando presentaban huevos en el ovario o embriones en el útero, o bien si la distensión del útero mostraba evidencia de anterior preñez. Los organismos con cicatriz umbilical parcialmente cerrada se consideraron “juveniles del año” (Carlson 1999). Para neonatos y juveniles del año, se verificó que presentaran algún resto de contenido estomacal. En el caso de hembras preñadas se contó el número de embriones y placentas; los embriones fueron medidos (Lt en $\mathrm{cm}$ ) y se les determinó el sexo.

\section{Descripción de unidades de pesca}

En la zona de estudio se encuentran tres unidades pesqueras que capturan tiburón, ya sea como pesca dirigida (tiburonera) o incidental (pelágica y demersal), cuyas características se presentan en la Tabla 1.

\section{Análisis de la información}

La estructura de tallas se obtuvo agrupando los datos en intervalos de $5 \mathrm{~cm}$ debido a la amplitud de las tallas (Branstetter 1987, Bonfil et al. 1993). La fecundidad se determinó por el conteo del número de embriones y placentas dentro del útero, a fin de considerar el caso de los embriones abortados durante la captura. El conteo de embriones a término es un indicador más adecuado del número de crías que sobrevivirán a la gestación. Para estimar el periodo de gestación, se graficó la longitud total de los embriones respecto de las fechas de muestreo (Pratt 1979, Parsons 1983, Yamaguchi et al. 1997).

Para determinar la talla de nacimiento y periodo de parto, se comparó la talla del embrión más grande dentro del útero, con la talla de los neonatos libres capturados a través del año (Pratt 1979, Francis \& Mace 1980, Conrath 2004).

La proporción de sexos se estimó por grupo ontogénico: embriones, neonatos, juveniles del año, juveniles y adultos y fue probada con un análisis de Chi cuadrado $(P<0,05)$ (Zar 1999). Se relacionó la presencia de embriones y juveniles del año con la temperatura promedio mensual, adquirida a partir de los registros contenidos en una base de datos en línea para TSM: http://iridl.ldeo.columbia.edu/SOURCES/.IGOSS/.nmc/. Reyn_SmithOIv1/.monthly/.sst/

Tabla 1

Descripción de las unidades de pesca utilizadas en la pesca artesanal de la costa de Oaxaca, México

Description of fishing units used in the artisan fishing in Oaxaca coast, Mexico

\begin{tabular}{|c|c|c|c|}
\hline Unidad de pesca & Especies objetivo & Equipos de pesca & Especies incidentales \\
\hline Demersal & Peces óseos demersales: & - Redes de enmalle & Tiburón (juveniles y neonatos) \\
\hline $\begin{array}{l}\text { Lanchas con motor fuera } \\
\text { de borda } 7,5 \mathrm{~m} \text { eslora/ } \\
\text { canoas de madera sin } \\
\text { motor }\end{array}$ & $\begin{array}{l}\text { Medregal (Caranx caballus) } \\
\text { Blanquito (Seriola sp.), } \\
\text { Roncadores, huachinango (Haemulon } \\
\text { flaviguttatum, H. maculicauda), } \\
\text { Pargo, (Lutjanus peru, Lutjanus guttatus, } \\
\text { L. argentiventis, L. colorado ), } \\
\text { Róbalo (Centropomus viridis, C. } \\
\text { robalito), etc. }\end{array}$ & - Cimbra de fondo & $\begin{array}{l}\text { Rayas (Dasyatis, Rhinobatos, } \\
\text { Gymnura), } \\
\text { tallas pequeñas de dorado } \\
\text { (Coryphaena hippurus) }\end{array}$ \\
\hline $\begin{array}{l}\text { Pelágica } \\
\text { Lanchas con motor fuera } \\
\text { de borda } 7,5 \mathrm{~m} \text { eslora }\end{array}$ & $\begin{array}{l}\text { Atún (Tunus albacares), } \\
\text { Barriletes (Euthynus linneatus, } \\
\text { Katsuwonus pelamis) }\end{array}$ & $\begin{array}{l}\text { - Curricanes } \\
\text { - Palangre de superficie } \\
\text { - Palangre de superficie } \\
\text { con boyas }\end{array}$ & $\begin{array}{l}\text { Tiburón (juveniles) } \\
\text { Picudos (Istiophorus platypterus, } \\
\text { Makaira mazara, } \text { M. indica), } \\
\text { Dorado (C. hippurus) }\end{array}$ \\
\hline $\begin{array}{l}\text { Tiburonera } \\
\text { Lanchas con motor fuera } \\
\text { de borda, } 10 \mathrm{~m} \text { eslora }\end{array}$ & $\begin{array}{l}\text { Tiburón (C. falciformis, C. limbatus, C. } \\
\text { leucas, S. lewini, A. pelagicus, } A \text {. } \\
\text { vulpinus, } N \text {. velox, M. lunulatus, C. } \\
\text { porosus, G. cuvier, R. lungurio) }\end{array}$ & $\begin{array}{l}\text { - Palangre de superficie } \\
\text { - Redes de deriva } \\
\text { - Palangre de superficie } \\
\text { con boyas }\end{array}$ & $\begin{array}{l}\text { Picudos (I. platypterus, M. mazara, } \\
\text { M. indica) } \\
\text { Dorado (C. hippurus) (tallas } \\
\text { grandes) }\end{array}$ \\
\hline
\end{tabular}




\section{Resultados}

Durante el periodo de muestreo se registraron 246 neonatos, 89 juveniles del año, 986 juveniles y 412 adultos correspondientes a C. falciformis y S. lewini, (Tabla 2), ya que ambas especies conforman el $75 \%$ de la pesca total de tiburón para la costa de Oaxaca. Los organismos fueron capturados por las tres unidades de pesca que operan en el área de estudio (Fig. 1).

\section{Sphyrna lewini}

Las capturas del tiburón martillo, S. lewini, estuvieron compuestas por 419 organismos, con tallas de 43 a 308 cm de Lt (Figs. 2 y 3). Durante julio y agosto en cada uno de los años de muestreo (TSM, 29-30 ${ }^{\circ} \mathrm{C}$ ), se registraron 184 neonatos (43,9\%) con tallas de 44 a 64 $\mathrm{cm}$ de Lt (talla promedio $=54 \mathrm{~cm}$ ) y una proporción de sexos 1:1 hembra:macho $\left(\chi^{2}=0,35 ; P>0,05\right)$. Los juveniles de año $(9,3 \%)$ que se reconocieron por tener la cicatriz umbilical parcialmente cerrada, se observaron durante agosto y septiembre, con tallas de 65 a $74 \mathrm{~cm}$ de Lt (talla media $=66 \mathrm{~cm}$ ) y una proporción de sexos 1:1 $\left(\chi^{2}=0,41 ; P>0,05\right)$ (Fig. 2). Los juveniles (127 organismos, 30,3\%) estuvieron presentes a partir de noviembre, con un intervalo de talla de 75 a $170 \mathrm{~cm}$ de Lt (talla media $=101 \mathrm{~cm}$ ), con una proporción de sexos de 2:1 hembra:macho $\left(\chi^{2}=13,9 ; P<0,05\right)$. Los adultos $(16,5 \%)$ con tallas de 170 a $308 \mathrm{~cm}$ de Lt y una proporción de sexos 1:3 hembra macho $\left(\chi^{2}=16,8\right.$; $P<0,05)$, se observaron en las capturas de diciembre a agosto (Fig. 3). Durante la temporada de lluvias se observó predominancia de hembras maduras.

Tabla 2

\section{Abundancia de los diferentes estadios de desarrollo ontogenético de $S$. lewini y $C$. falciformis en función de la unidad de pesca utilizada en la costa de Oaxaca}

Abundance of different ontogenetic development stages of S. lewini and C. falciformis, based on fishing unit used in the coast of

Oaxaca

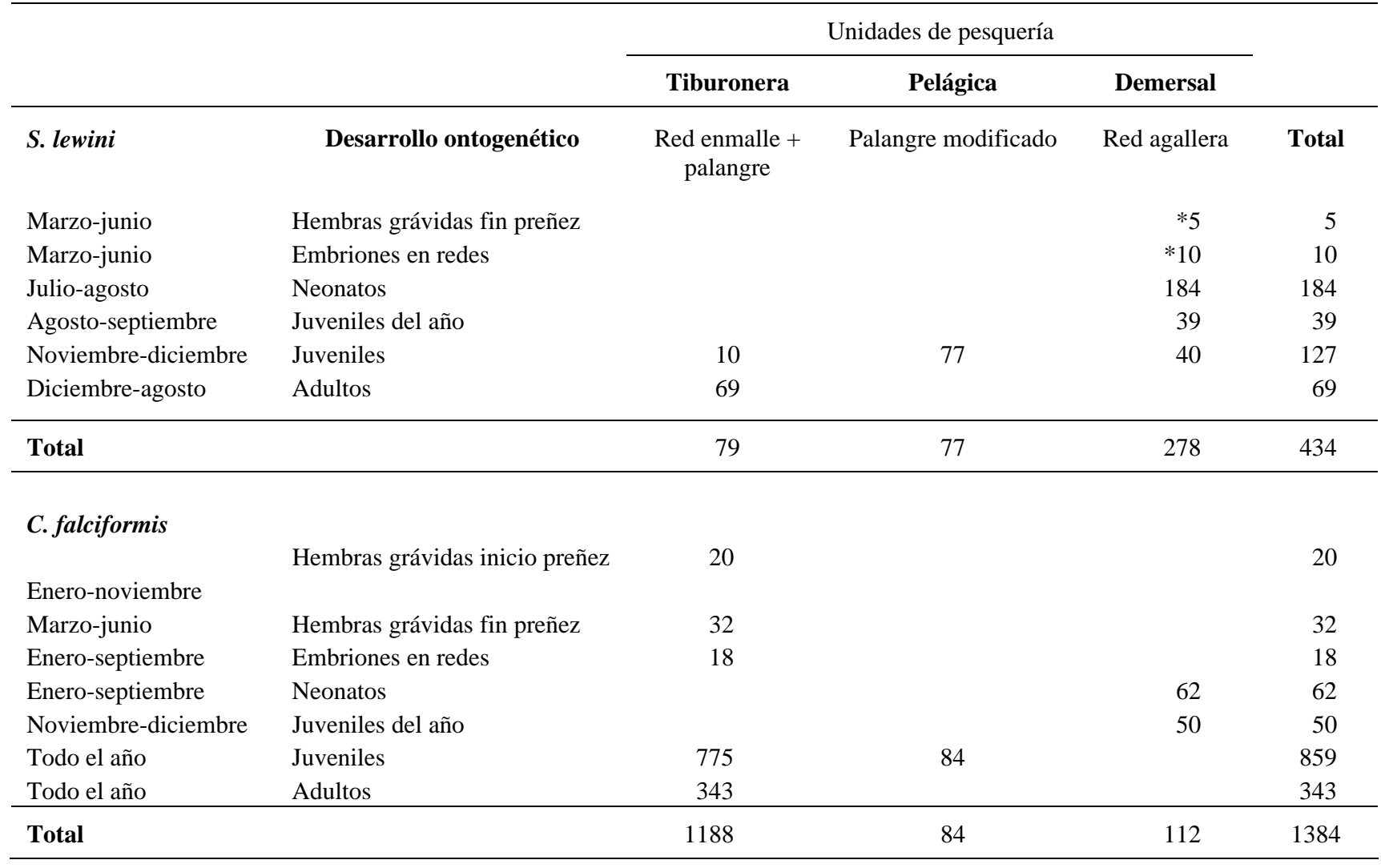

* Sólo se presentaron en 2003 


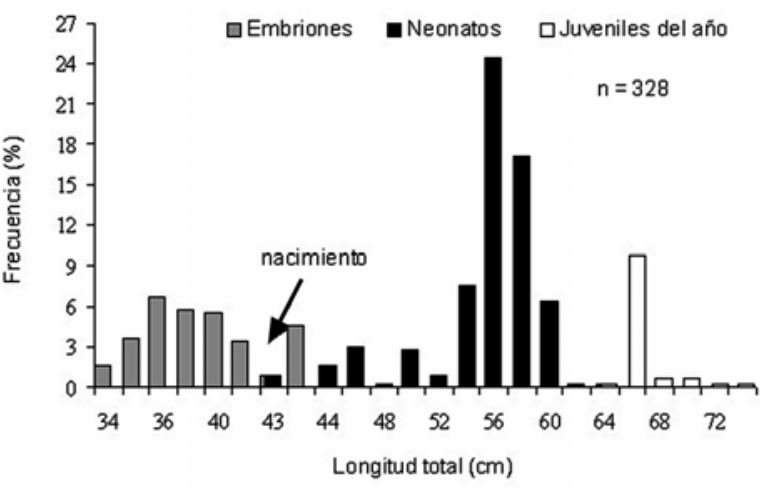

Figura 2

Distribución de frecuencia de la longitud de embriones, neonatos y juveniles del año de $S$. lewini en las capturas artesanales de la costa de Oaxaca, México

Length frequency distribution of embryos, newborns and young of the year of $S$. lewini caught in artisan fishery of the Oaxaca coast, Mexico

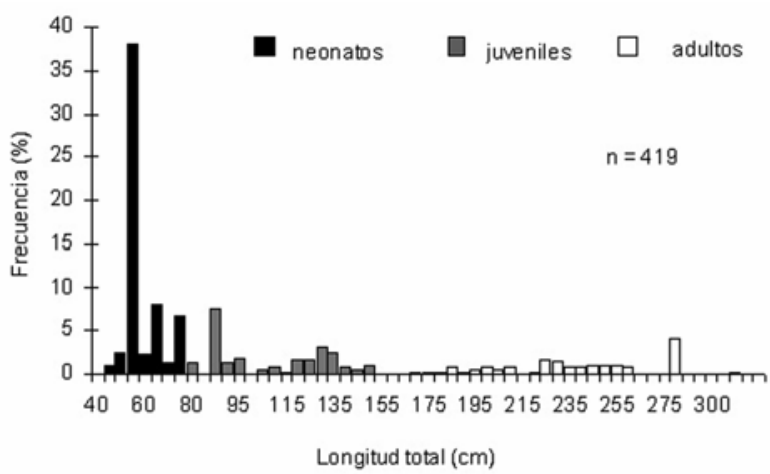

Figura 3

Distribución de frecuencia de la longitud de neonatos, juveniles y adultos de $S$. lewini en las capturas artesanales de la costa de Oaxaca, México

Length frequency distribution of newborns, young and adults of $S$. lewini caught in artisan fishery of the Oaxaca coast, Mexico

Tabla 3

Resumen de los meses de captura, longitud total (Lt), número y proporción de sexos de embriones encontrados en cinco hembras grávidas de $S$. lewini

Summary of the month of capture, total length (Lt), number and sex ratio of embryos registered in five pregnant females of $S$. lewini

\begin{tabular}{lcccc}
\hline \multicolumn{1}{c}{ Fecha } & $\begin{array}{c}\text { Lt hembra } \\
(\mathrm{cm})\end{array}$ & $\mathrm{N}^{\text {o }}$ de embriones & $\begin{array}{c}\text { Rango de Lt } \\
\text { embriones (cm) }\end{array}$ & $\begin{array}{c}\text { Proporción sexos } \\
\text { (hembra-macho) }\end{array}$ \\
\hline 15 abril 2003 & 160 & 24 & $35-38$ & $1: 1$ \\
17 mayo 2003 & 160 & 18 & $40-45$ & $1: 1$ \\
17 mayo 2003 & 165 & 20 & $40-43$ & $1: 1$ \\
17 mayo 2003 & 170 & 23 & $40-45$ & $1: 1$ \\
06 junio 2003 & 160 & 20 & $38-40$ & \\
\hline
\end{tabular}

En marzo, mayo y junio de 2003 se registraron cinco hembras grávidas con tallas entre los 160 a 165 cm de Lt. La fecundidad de estas hembras varió entre 18 y 24 embriones por hembra (media $=21$ embriones), con una talla de 35 a $45 \mathrm{~cm}$ de Lt (talla media $=40 \mathrm{~cm}$ ) y una proporción de sexos 1:1 $\left(\chi^{2}=0,51 ; P>0,05\right)$. (Tabla 3). Debido al reducido número de hembras grávidas observadas, se estimó de manera preliminar la talla de nacimiento en $43 \mathrm{~cm}$ de Lt, ya que el embrión más grande midió $45 \mathrm{~cm}$ y el neonato más chico $43 \mathrm{~cm}$ de Lt. De acuerdo a la frecuencia de neonatos, se plantea que el periodo de parto ocurre entre julio y agosto.

\section{Carcharhinus falciformis}

Durante el periodo de muestreo, el tiburón sedoso $C$. falciformis se presentó como la especie con mayor abundancia en las capturas de tiburón. Se registraron un total de 1314 individuos, con tallas de 65 a $320 \mathrm{~cm}$ de 


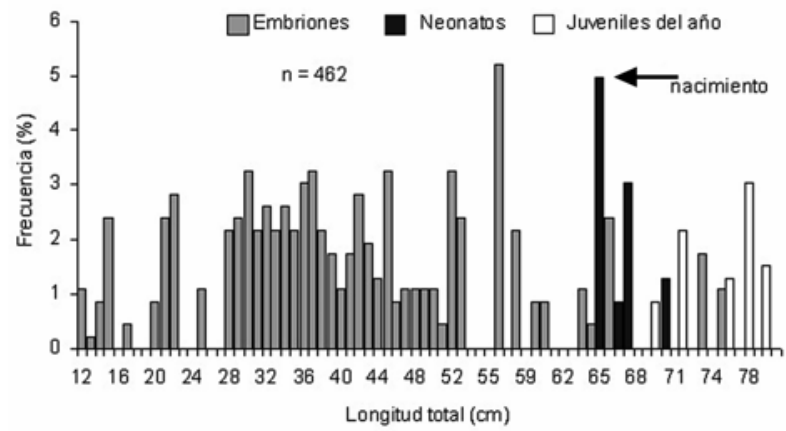

Figura 4

Distribución de frecuencia de la longitud de embriones, neonatos y juveniles del año de $\boldsymbol{C}$. falciformis en las capturas artesanales de la costa de Oaxaca, México

Length frequency distribution of embryos, newborns and young of the year of $C$. falciformis caught in artisan fishery of the Oaxaca coast, Mexico

Lt (Figs. 4 y 5). Durante todos los años del muestreo se registraron 62 neonatos $(4,7 \%)$, que formaron parte de la pesca incidental de la unidad demersal, mismos que se presentaron en los meses de enero a septiembre, con tallas de 65 a $71 \mathrm{~cm}$ de Lt (talla media $=65 \mathrm{~cm}$ ) y una proporción sexual de 1:1 hembra:macho $\left(\chi^{2}=0,14\right.$; $P>0.05$ ). Los juveniles del año (50 organismos, 3.8\%) se presentaron en las capturas de la unidad demersal en el mes de agosto, con tallas de 72 a $80 \mathrm{~cm}$ de Lt (talla media $=75 \mathrm{~cm})$ y una proporción de sexos de $1: 1\left(\chi^{2}=\right.$ 1,62; $P>0,05$ ) (Fig. 4). Se registraron 859 juveniles $(65,4 \%)$, con un intervalo de talla de 80 a $170 \mathrm{~cm}$ de $\mathrm{Lt}$ (talla media $=127 \mathrm{~cm}$ ) y con una proporción de sexos de $1: 1\left(\chi^{2}=0,38 ; P>0,05\right)$; estos estuvieron presentes en las capturas todo el año, siendo más abundantes al final de la época de secas (marzo-abril). El 26,1\% correspondió a los adultos, con tallas de 171 a $320 \mathrm{~cm}$ de Lt y una proporción de sexos 1:1 $\left(\chi^{2}=0,75\right.$; $P>0,05$ ) (Fig. 5).

A lo largo del periodo de estudio, en los meses de enero a noviembre, se registraron en las capturas 52 hembras grávidas de C. falciformis con un intervalo de longitud total de 169 a $256 \mathrm{~cm}$ (Fig. 6) y con fecundidad de 3 a 14 embriones por hembra; la longitud total de los embriones varió de 10 a $66 \mathrm{~cm}$, con una proporción de sexos de 1:1,2 hembra-macho $\left(\chi^{2}=2,58 ; P>0,05\right)$. Así

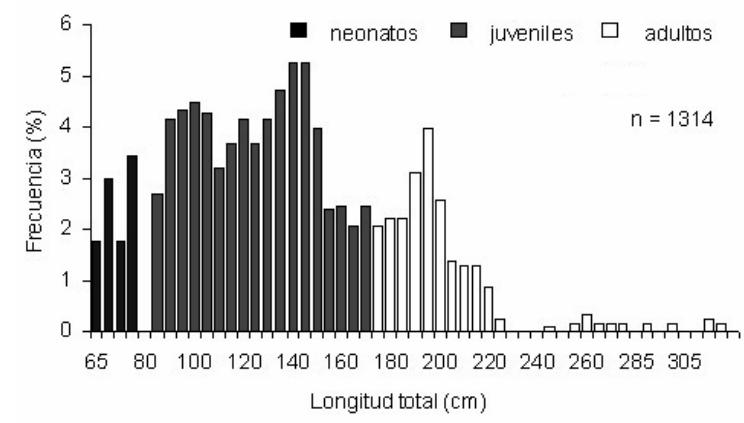

Figura 5

Distribución de frecuencia de la longitud de neonatos, juveniles y adultos de $\boldsymbol{C}$. falciformis en las capturas artesanales de la costa de Oaxaca, México

Length frequency distribution of newborns, young and adults of $C$. falciformis caught in artisan fishery of the Oaxaca coast, Mexico

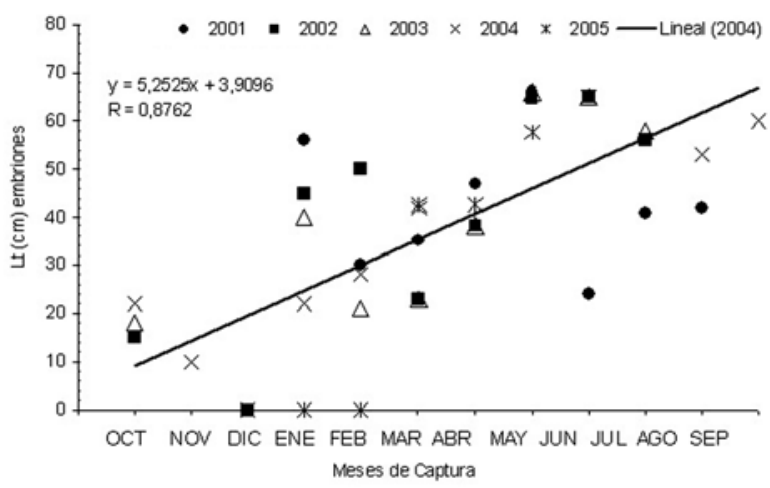

Figura 6

Relación entre la longitud de los embriones de $C$. falciformis y sus capturas por mes y año, en la costa de Oaxaca, México. $\mathbf{N}=\mathbf{3 5 0}$

Embryo length capture relationship by month and year of $C$. falciformis, for Oaxaca coast, Mexico. $\mathrm{N}=350$

mismo, se observaron embriones en diferentes estados de desarrollo en las redes de pesca, lo cual indica aborto durante la captura debido al trauma de las hembras al luchar para liberarse de las redes.

La distribución de frecuencia-longitud de embriones y neonatos indica que la talla de nacimiento es de 65 


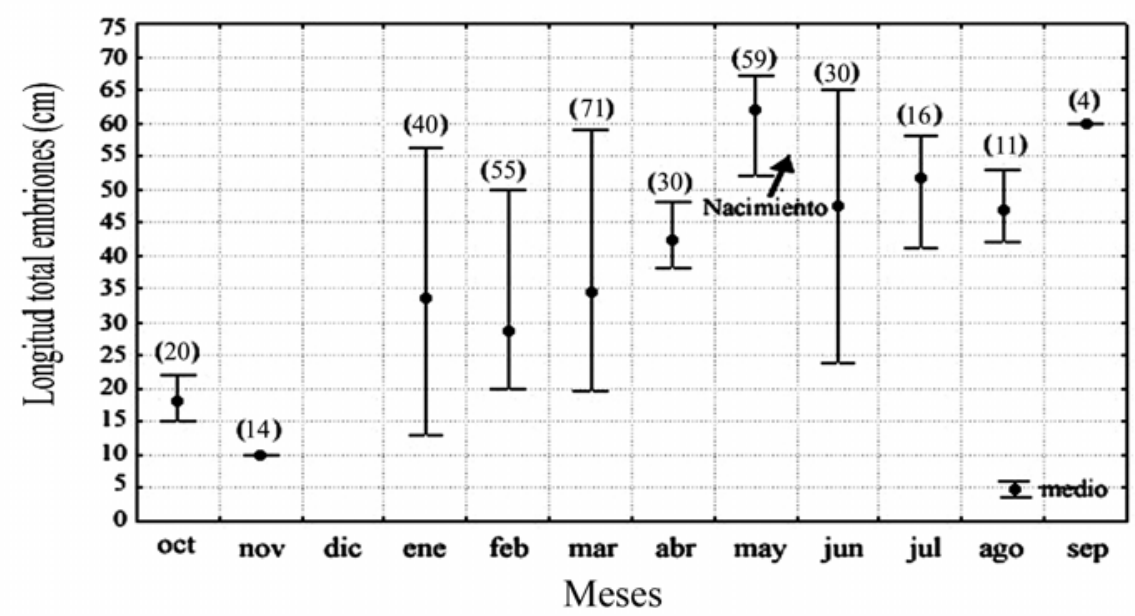

Figura 7

Desarrollo de embriones de $C$. falciformis a través del tiempo. En paréntesis: número total de embriones

Development of $C$. falciformis through time. In parentheses: total embryos number

cm de Lt, ya que el embrión más grande midió 66 cm y el neonato más pequeño $65 \mathrm{~cm}$ de Lt. Se plantea un periodo de gestación de aproximadamente 12 meses, con nacimientos a lo largo del año, con dos picos reproductivos, el primero de enero a marzo y uno mayor de mayo a septiembre, en tanto que de octubre a diciembre se registraron únicamente embriones pequeños (Fig. 7).

\section{Otras especies}

En septiembre y octubre de 2004, se observaron 18 neonatos de $C$. limbatus, con una talla de 62 a $85 \mathrm{~cm}$ de Lt, así como 8 juveniles con una Lt de 86 a 125 cm. En agosto se observaron en las capturas demersales dos hembras y un macho maduros. Durante la temporada de lluvias la unidad de pesca demersal capturó 5 neonatos de C. porosus, 3 de Nasolamia velox y 5 de Mustelus lunulatus.

\section{Discusión}

En la costa de Oaxaca la pesca de tiburón es artesanal y multiespecífica, con una alta dependencia estacional. Los juveniles y adultos prereproductivos del tiburón sedoso, C. falciformis, fueron los más abundantes en las capturas de la unidad pelágica y tiburonera, por lo tanto sostienen la pesquería durante la mayor parte del año. Sin embargo, el tiburón martillo, S. lewini, se capturó con mayor abundancia en la época de lluvias, lo cual obedece también, a que durante esta temporada el esfuerzo aplicado se centra en la pesca demersal. Debido a que se desarrolla con embarcaciones pequeñas y reducido radio de operación, es frecuente la captura incidental de neonatos y juveniles del tiburón martillo, así como ocasionales adultos de tallas reproductivas y hembras grávidas.

Los organismos maduros y juveniles, así como algunas hembras grávidas en etapas iniciales de preñez se observaron en las capturas de la unidad tiburonera. Debido a que la unidad de pesquería pelágica opera más cerca de la costa registró las mayores capturas de juveniles, así como hembras grávidas a término; los neonatos y juveniles del año forman parte de la pesca incidental de la unidad demersal.

Los resultados del presente trabajo son equiparables a la región de Puerto Madero, Chiapas, donde la pesquería de tiburón es soportada principalmente por $C$. falciformis y S. lewini, en la que aproximadamente $80 \%$ de la captura corresponde a organismos juveniles ${ }^{1}$. Para la costa michoacana varios autores (Ruíz 1983, Madrid et al. 1997, Anislado 2000) reportan que la pesca de

\footnotetext{
${ }^{1}$ Soriano-Velázquez S, DE Acal, CT Galvan, J Castillo-Geniz, C Ramírez-Santiago \& F Sancho-Vázquez. 2002. Aspectos reproductivos de tres especies de la familia Carcharhinidae y una especie de la familia Sphyrnidae del Golfo de Tehuantepec. Memorias del VIII Congreso Nacional de Ictiología, Puerto Ángel, Oaxaca, México, p. 58.
} 
tiburón es sostenida por neonatos y juveniles de $S$. lewini.

La presencia considerable de juveniles en las capturas incidentales y dirigidas a tiburón puede estar generando un desequilibrio poblacional de estas especies en la costa Pacífica, ya que el porcentaje de captura es muy alto como para poder garantizar la sostenibilidad de las especies en el tiempo. Lo anterior ha sido observado en el Golfo de México, donde la falta de control sobre las pesquerías y la fragilidad biológica de las especies de tiburón ha ocasionado una considerable disminución de la abundancia de las especies (Bonfil 1997).

Respecto a la época y talla de nacimiento, los cambios en la longitud total de 350 embriones de $C$. falciformis en 52 camadas, indican un periodo de gestación de aproximadamente doce meses, con dos picos reproductivos a lo largo del año. Para el primer grupo, la temporada de nacimientos se encuentra relacionada con los vientos tehuanos, que ocurren de octubre a marzo (Trasviña \& Barton 1997). Para el segundo grupo, el inicio de la época de lluvias marca la temporada de nacimientos, lo cual ocurre a partir de mayo y se extiende hasta septiembre. Branstetter (1987) y Bonfil et al. (1993) indican que la época de reproducción de $C$. falciformis en aguas templado cálidas ocurre desde junio a agosto y en los trópicos no tiene un periodo de gestación estacionario, por lo que el nacimiento puede darse en periodos alternos. Al respecto, Pratt \& Casey (1990) mencionan que la temporada de gestación y apareamiento dependen principalmente de la temperatura.

La talla de nacimiento estimada en este estudio (65 cm de Lt) es menor a la reportada para otras regiones, en el banco de Campeche. Bonfil et al. (1993) señalan una talla de expulsión de $76 \mathrm{~cm}$ de Lt; en el Golfo de California, Cadena-Cárdenas (2001) menciona una talla de $70 \mathrm{~cm}$ de Lt y para el Golfo de Tehuantepec de 57 a $77 \mathrm{~cm}$ de $\mathrm{Lt}^{1}$. Lo anterior puede estar correlacionado con la temperatura superficial del mar (TSM), que durante julio y agosto alcanza los valores más altos del año $\left(28-30^{\circ} \mathrm{C}\right)$.

En la zona de estudio se observó un número importante de hembras en etapas iniciales de preñez, en comparación con lo reportado por otros trabajos en la región norte del Pacífico mexicano (Castillo 1992, Cadena-Cárdenas 2001) donde reportan hembras grávidas en estado intermedio y avanzado de preñez.
Holden (1974) menciona que las hembras después de la cópula y en las etapas iniciales de preñez se mueven hacia áreas fuera de la plataforma continental y de acuerdo a Springer (1967), esta migración está influenciada por la temperatura superficial del mar. Sin embargo, la angostura de plataforma continental en la costa chica de Oaxaca y las características oceanográficas de la zona, pueden explicar la presencia de hembras grávidas de $C$. falciformis en las capturas artesanales en etapas iniciales de preñez.

Para S. lewini, Branstetter (1990) menciona una talla promedio de los neonatos mayor a $50 \mathrm{~cm}$ de Lt en la región del Atlántico noroeste; Anislado (2000) para el Pacífico centro señala la época de parto de mayo a agosto, con una talla promedio de $46,5 \mathrm{~cm}$ de Lt. La talla estimada en el presente trabajo (45 cm de Lt) es muy cercana a la reportada por Anislado (2000). Durante el periodo de muestreo, en los meses de julio y agosto, en las capturas incidentales de la unidad demersal se observó un número importante de neonatos y juveniles, que bien puede corresponder al pico de reproducción. Al respecto, diversos autores mencionan que los neonatos y juveniles del tiburón martillo normalmente se encuentran en zonas costeras, cerca del litoral, formando grandes cardúmenes (Compagno 1984, Hazin et al. 2001, Anislado 2000) y además, debido a la forma de su cabeza, son muy susceptibles de capturar con las redes de enmalle (Wakabayashi 1981, Klimley 1987).

Las crías de $S$. lewini nacen aproximadamente al mismo tiempo que el segundo grupo de C. falciformis; esto ocurre durante la temporada de lluvias, cuando se presentan las temperaturas más elevadas del año y se encuentra una alta disponibilidad de alimento en el litoral oaxaqueño (Pennington et al. 2006). Al respecto, Branstetter (1990), Castro (1993) y Simpfendorfer \& Milward (1993) mencionan que los neonatos tienen altas tasas de crecimiento, lo que indica un consumo elevado de alimento por lo que tienden a permanecer en áreas donde el alimento es abundante. El uso de un área de expulsión común entre dos o más especies ha sido reportado para otras regiones, Babler et al. (1989), Salini et al. (1992) y Simpfendorfer \& Milward (1993) en el norte de Australia; Castro (1993) en la bahía Bulls en Carolina del Sur, EUA; Castillo et al. (1998) para Veracruz y Tabasco y Anislado (2000) en Michoacán, México. Así mismo, se ha reportado que el uso de un área de crianza por varias especies, puede ser ventajoso para reducir la depredación (Simpfendorfer \& Milward 1993). 
Por otra parte, la región sur del Golfo de Tehuantepec es considerada como un área de agregación de hembras grávidas de S. lewini; sin embargo, en las capturas artesanales de la costa chica de Oaxaca únicamente se presentan algunos adultos maduros y hembras preñadas a término. Varios autores (Compagno 1984, Branstetter 1987, Chen et al. 1988, Last \& Stevens 1994) mencionan que $S$. lewini presenta una dependencia estrecha al movimiento de corrientes cálidas, para penetrar en bahías y estuarios para su apareamiento y parto. Además, se ha documentado que esta especie realiza movimientos horizontales y verticales en busca de alimento (Klimley et al. 1993, Anislado 2000). Lo anterior puede ayudar a explicar por qué en las capturas de esta zona se encuentran hembras en avanzado estado de preñez, lo cual es posible al ser atraídas a la costa por la disponibilidad de alimento. La poca información sobre hembras y en particular sobre hembras grávidas, se puede atribuir a la segregación sexual de los adultos y a la preferencia de las hembras por áreas más profundas, las que se ubican lejos de las zonas de pesca costera (Branstetter 1987, Stevens \& Lyle 1989).

En general, las áreas de crianza son hipotéticas (Castro 1993), las hembras grávidas, recién nacidos y juveniles permanecen en las áreas de crianza las primeras semanas, meses o años. Springer (1967) sugiere que los depredadores importantes de tiburón son otros tiburones y que las áreas de crianza pueden ser cerradas, debido a la ausencia de grandes tiburones. Para la costa chica de Oaxaca, el registro de hembras grávidas en avanzado estado de desarrollo en las capturas pelágicas a no más de 10 millas de la costa y la presencia de neonatos y juveniles del año en las redes de enmalle, sugiere que en la zona de pesca de estas embarcaciones, frecuentemente cerca de las bocabarras de los ríos Copalita, Tonameca y Río Grande, así como en las inmediaciones de las bahías de Pto. Ángel y Tagolunda, existe un área de alumbramiento y crianza de $C$. falciformis y $S$. lewini.

Carcharhinus falciformis y Sphyrna lewini son dos especies cosmopolitas pelágico-costeras, con una importante captura en ambos litorales de México (Soriano et al. 2006). Al mismo tiempo, debido a la alta presión de pesca sobre las crías del tiburón martillo, se le ha incluido en la lista de las 26 especies de tiburón con prioridad de conservación (Chen et al. 1988). Respecto a C. falciformis, Bonfil et al. (1993) menciona que de acuerdo a las características de su historia de vida, es un recurso muy frágil, el cual no puede soportar una elevada presión de pesca. Lo anterior, resalta la importancia de realizar estudios enfocados a definir las áreas de expulsión y crianza en aguas del centro y sur del Pacífico mexicano, así como las vías de migración que podrían necesitar protección especial.

De los componentes de las poblaciones, los más impactados son los recién nacidos (neonatos) y juveniles, por lo que su pesca intensiva puede provocar un desequilibrio en la tasa de renovación de estas especies y generar un colapso pesquero (Musick et al. 1993). Dado lo anterior, la protección de las áreas de crianza es fundamental en la conservación de los tiburones y deberá figurar en todo plan de manejo de estas especies, debido a que la mortalidad por pesca es extremadamente alta en estas áreas y la dinámica del stock recluta es afectada (Bonfil 1997).

\section{Agradecimientos}

A los pescadores de la costa de Oaxaca por las facilidades en el registro de datos. A los estudiantes adscritos al Laboratorio de Ictiología y Biología Pesquera de la UMAR por su colaboración en el trabajo de campo. A los árbitros anónimos por sus revisiones y comentarios. El presente estudio fue financiado por la Universidad del Mar (clave: 2IR0404).

\section{Literatura citada}

Alejo-Plata MC, GLG Cerdenares \& GM González. 2006a. La pesca de tiburón en la costa chica de Oaxaca, México, 2000-2003. En: Salas S, MA Cabrera, J Ramos, D Flores \& J Sánchez (eds). Memorias Primera Conferencia de Pesquerías Costeras en Latinoamérica y el Caribe. Evaluando, Manejando y Balanceando Acciones, pp. 22-38. EPOMEX, México.

Alejo-Plata MC, SC Ramos \& JLR Cruz. 2006b. La pesquería artesanal del tiburón en Salina Cruz, Oaxaca, México. Ciencia y Mar 30: 37-51.

Anislado TV. 2000. Ecología pesquera del tiburón martillo Sphyrna lewini (Griffith y Smith, 1834) en el litoral del estado de Michoacán, México. Tesis de Maestría en Ciencias, Facultad de Ciencias, Universidad Nacional Autónoma de México, México, 115 pp.

Babler SJM, SW Young \& MC Dunning. 1989. Species composition and biomass of fishes in different habitats of a tropical northern Australian estuary: their occurrence in the adjoining sea and estuarine dependence. Estuarine, Coastal and Shelf Science 29: 509-531. 
Bonfil R, $R$ Mena \& $D$ de Anda. 1993. Biological parameters of commercially exploited silky sharks, Carcharhinus falciformis, from the Campeche Bank, Mexico. NOAA Technical Reports NMFS 115: 73-86.

Bonfil SR. 1997. Status of shark resources in the southern Gulf of Mexico and Caribbean. Implications for management. Fisheries Research 29: 101-117.

Branstetter S. 1987. Age, growth and reproductive biology of the silky shark, Carcharhinus falciformis, and the scalloped hammerhead, Sphyrna lewini, from the northwestern Gulf of Mexico. Environmental Biology of Fishes 19(3): 161-173.

Branstetter S. 1990. Early life-history implications of selected carcharhinoid and lammoid sharks of the northwest Atlantic. NOAA Technical Reports NMFS 90: 17-28.

Cadena-Cárdenas L. 2001. Biología reproductiva de Carcharhinus falciformis (Chondrichthyes: Carcharhiniformes: Carcharhinidae), en el Golfo de California. Tesis de Biología Marina, Universidad Autónoma de Baja California Sur, México, 68 pp.

Carlson JK. 1999. Ocurrence of neonato and juvenile sandbar sharks, Carcharhinus plumbeus, in the northeastern Gulf of Mexico. Fishery Bulletin 97: 387391.

Carlson JK \& JH Brusher. 1999. An index of abundance for coastal species of juvenile sharks from the Gulf of Mexico. Marine Fisheries Review 61(3):37-45.

Castillo GJL. 1992. Diagnóstico de la pesquería del tiburón en México, 76 pp. Instituto Nacional de la Pesca, Secretaria de Pesca, México.

Castillo GJL, JF Márquez-Farias, MC Rodríguez de la Cruz \& A Cid del Prado. 1998. The Mexican artisanal shark fishery in the Gulf of Mexico: towards a regulated fishery. Marine and Freshwater Research 49: 611-620.

Castro JI. 1983. The sharks of North American waters, 179 pp. Texas A \& M University Press, College Station, Texas.

Castro JI. 1993. The nursery of Bull Bay, South Carolina, with a review of the shark nurseries of the Southeastern coast of the United States. Environmental Biology of Fishes 38: 37-48.

Castro JI. 2000. Guía para la identificación de las especies de tiburones de importancia comercial en el Océano Pacífico, 4 pp. SAGARPA. Subsecretaría de Pesca. Instituto Nacional de la Pesca, México.

Chen CT, TC Leu \& J Joung 1988. Reproduction in the female scalloped hammerhead, Sphyrna lewini, in Northeastern Taiwan waters. Fishery Bulletin 86(2): 389393.
Compagno JVL. 1984. Sharks of the world. An annotated and illustrated catalogue of shark species known to date. Part 2: Carcharhiniformes FAO Fisheries Synopsis No 125, Vol. 4, Part 2: 1-665.

Compagno LJV, F Krupp \& W Schneider. 1995. Tiburones. En: Fisher W, F Krupp, W Schneider, C Somer, KE Carpenter \& VH Niem (eds). Guía FAO para la identificación de especies para los fines de pesca. Pacífico Centro-Oriental, 2: 648-743. Food and Agriculture Organization of the United Nations, Roma.

Conrath LC \& JA Musick. 2007. Investigations into depth and temperature habitat utilization and overwintering grounds of juvenile sandbar sharks, Carcharhinus plumbeus: the importance of near shore North Carolina waters. Environmental Biology of Fishes 79: 137-154.

Conrath LC. 2004. Reproductive Biology. En: Musick JA \& $\mathrm{R}$ Bonfil (eds). Elasmobranch Fisheries Management Techniques, pp. 133-164. Asia-Pacific Economic Cooperation (APEC) Fisheries Working Group, Singapore.

Costantini M \& M Affronte. 2003. Neonatal and juvenile sandbar shark in the northern Adriatic Sea. Journal of Fish Biology 62: 740-743.

Francis MP \& JT Mace. 1980. Reproductive biology of Mustelus lenticulatus from Kaikoura and Nelson. New Zealand Journal of Marine Freshwater Research 14: 303311.

Garrick JAF. 1982. Sharks of the genus Carcharhinus. NOAA Technical Report NMFS Circular 445: 1-194.

Grace MA. 2001. Field guide to requiem sharks (Elasmobranchiomorphi: Carcharhinidae) of the Western North Atlantic. NOAA Technical Report NMFS 153: 132.

Hazin F, A Fisher \& M Broadhurst. 2001. Aspects of reproductive biology of the scalloped hammerhead shark, Sphyrna lewini, off northeastern Brazil. Environmental Biology of Fishes 61: 151-159.

Holden MJ. 1974. Problems in the rational exploitation of elasmobranches populations and some suggested solutions. En: Harden-Jones FR (ed). Sea Fisheries Research, pp. 113-137. John Wiley and Sons, Nueva York.

Klimley AP. 1987. The determinants of sexual segregation in the scalloped hammerhead Sphyrna lewini. Environmental Biology of Fishes 18: 27-40.

Klimley AP, I Cabrera-Mancilla \& LG Castillo. 1993. Descripción de los movimientos horizontales y verticales del tiburón martillo Sphyrna lewini del sur del Golfo de California, México. Ciencias Marinas 19 (1): 95-115. 
Last PR \& JD Stevens. 1994. Sharks and Rays of Australia, 513 pp. Commonwealth Scientific and Industrial Research Organization, Australia.

Madrid VJ, P Sánchez \& AL Ruiz. 1997. Diversity and abundance of a tropical fishery on the Pacific shelf of Michoacan, México. Estuarine Coastal and Shelf Science 45: 485-495.

Merson RR \& HL Pratt Jr. 2001. Distribution, movements and growth of young sanbar sharks, Carcharhinus plumbeus, in the nursery grounds of Delawere Bay. Environmental Biology of Fishes 61: 13-24.

Musick JAS, S Branstetter \& JA Colvocoresses. 1993. Trends in shark abundance from 1974 to 1991 for the Chesapeake Bight region of the US. mid Atlantic coast. NOAA Technical Report NMFS Circular 115:1-18.

Parsons GR. 1983. The reproductive biology of the Atlantic sharpnose shark Rhizoprionodon terranovae (Richardson). Fishery Bulletin 81: 61-73.

Pennington TJ, LM Kevin-Kuwahara, VS Kolber, DD Calientes \& PF Chavez. 2006. Primary production in the eastern tropical Pacific: A review. Progress in Oceanography 69: 287-17.

Pratt HL. 1979. Reproduction in the blue shark, Prionace glauca. Fishery Bulletin 77: 445-470.

Pratt, Jr HL \& JG Casey. 1990. Shark reproductive strategies as limiting factor in direct fisheries, with a review of Holden's method of estimating growthparameter. NOAA Technical Report NMFS 90: 97-108.

Ruíz LA. 1983. Contribución al conocimiento de los peces marinos de importancia comercial en Bahía Bufadero, Michoacán, México. Tesis de Licenciatura, Universidad Nacional Autónoma de México, México, 137 pp.

Salini JP, SJM Blazer \& DT Brewer. 1992. Diets of sharks from estuaries and adjacent waters of the north-eastern Gulf of Carpentaria, Australia. Australian Journal of Marine Freshwater Research 43: 87-96.
Saucedo CJB, G Colado, JG Martínez, S Burgos, JG Chacon \& J Espinoza. 1982. Contribución al estudio de la pesquería del tiburón en la zona del estado de Sinaloa. Memoria de Servicio Social, Escuela de Ciencias del Mar, Universidad Autónoma de Sinaloa, México, 60 pp.

Simpfendorfer CA \& NE Milward. 1993. Utilization of a tropical bay as a nursery area by sharks of the families Carcharhinidae and Sphyrnidae. Environmental Biology of Fishes 37: 337-345.

Soriano VS, ED Acal, CL Castillo, VN Gómez \& RC Santiago. 2006. Tiburones del Golfo de Tehuantepec. En: Arreguín SF, MJ Meléndez, MI Gómez-Humarán, SR Solana \& DC Rangel (eds). Sustentabilidad y pesca responsable en México, Evaluación y Manejo 1999-2000, pp. 325-364. Instituto Nacional de la Pesca, México.

Springer S. 1967. Social organization of shark populations. En: Mathewson PW \& DP Rall (eds). Sharks, skates and rays, pp.149-174. John Hopkins Press, Baltimore.

Stevens JD \& JM Lyle. 1989. Biology of three hammerhead sharks (Eusphyra blochii, Sphyrna mokarran and $S$. lewini) from northern Australia. Australian Journal of Marine and Freshwater Research 40: 129-146.

Trasviña A \& ED Barton. 1997. Los Nortes del Golfo de Tehuantepec: Dinámica del océano costero. En: Lavin M (ed). Contribuciones a la Oceanografía Física en México. Monografía $\mathrm{N}^{\mathrm{o}}$ 3: 25-46. Unión Geofísica Mexicana, México.

Wakabayashi K \& T Iwanmoto. 1981. Schooling of the scalloped hammerhead Sphyrna lewini, in the Gulf of California. U.S. Fishery Bulletin 79: 356-359.

Yamaguchi A, T Taniuchi \& M Shimizu. 1997. Reproductive biology of starspotted dogfish Mustelus manazo from Tokyo Bay, Japan. Fisheries Science 63(6): 918-922.

Zar JH. 1999. Biostatistical analysis, 663 pp. Prentice Hall, Englewood Cliffs.

Recibido el 30 de octubre de 2006 y aceptado el 24 de septiembre de 2007 